\title{
La physiopathologie de l'hypertension artérielle en dialyse chronique
}

\section{The physiopathology of dialysis-associated hypertension}

\author{
E. P. Cohen ${ }^{a^{*}}$, J.-M. Krzesinski ${ }^{b}$
}

aDepartment of Medicine, Froedtert Hospital - Medical College of Wisconsin, 9200 W Wisconsin Ave, Milwaukee, Wisconsin, USA 53226, États-Unis

bService de Néphrologie, CHU de Liège - Université de Liège, 4000 Liège, Belgique

\section{MOTS CLÉS}

Hypertension ;

Dialyse chronique ;

Volume extracellulaire ;

Résistances artérielles ;

Sodium du dialysat ;

Efficacité dialytique ;

Système nerveux

sympathique ;

Rénine-angiotensine ;

Endothéline ;

Oxyde nitrique ;

Érythropoiétine

\section{KEYWORDS}

Hypertension;

Chronic dialysis;

Extracellular volume;

Arterial resistance;

Dialysate sodium;

Dialysis delivery;

Sympathetic nervous

system;

Renin-angiotensin;

Endothelin;

Nitric oxide;

Erythropoietin
Résumé L'hypertension chez le sujet en dialyse chronique est fréquente. Ses origines se trouvent dans la surcharge du volume extracellulaire, compliquée par l'augmentation des résistances artérielles. Ces dernières sont affectées par diverses systèmes, dont la rénineangiotensine, l'endothéline, l'oxyde nitrique, le système nerveux orthosympathique et d'autres. L'interaction entre tous ces facteurs peut expliquer que le contrôle de l'hypertension en dialyse requiert une attention constante aux multiples aspects d'un traitement dialytique efficace.

(c) 2007 Elsevier Masson SAS. Tous droits réservés.

Abstract Hypertension in subjects on long term dialysis is frequent. Its origins are found in extracellular volume overload, which is complicated by increased peripheral arterial resistance. The latter is affected by many systems, including that of renin-angiotensin, endothelin, nitric oxide, the sympathetic nervous system, and others. The interaction between these factors may explain why the control of hypertension in dialysis patients requires ongoing attention to the many aspects of good dialysis. (c) 2007 Elsevier Masson SAS. Tous droits réservés. 


\section{Introduction}

Les origines de l'hypertension artérielle (HTA) essentielle font l'objet de maintes études, sans qu'une physiopathologie unique ait pu être précisée. Chez le sujet en dialyse chronique, l'HTA est encore plus fréquente, mais sa physiopathologie a été moins souvent investiguée. Pourtant, le manque de contrôle de la tension artérielle (TA) est une réalité chez beaucoup de patients en dialyse chronique. Une meilleure compréhension de la physiopathologie pourrait combler ce déficit.

Dans cet article, nous prendrons en considération la TA avant la dialyse, moment où ce paramètre est souvent le plus élevé.

Il est établi que la fréquence de l'HTA augmente avec la diminution de la fonction rénale pour culminer lorsque le patient entre en dialyse chronique [1]. En effet, près de $90 \%$ des patients à ce stade ont une TA systolique en prédialyse supérieure à $150 \mathrm{mmHg}$ [2]. Cette HTA est améliorée mais non éliminée par le traitement dialytique.

La physiopathologie de l'HTA du patient dialysé n'est pas seulement une question de volume extracellulaire $[\mathrm{VEC}]$. D'autres facteurs ont été incriminés dont une élévation des résistances artérielles périphériques et une altération des grosses artères. Cette HTA est souvent systolo-diastolique ou peut-être encore plus fréquemment systolique isolée [3]. On peut identifier les facteurs reconnus (Tableau 1).

\section{Analyse de travaux récents}

Dans cette mise au point, nous avons centré notre recherche sur "Medline", prenant comme mots clés «hypertension/pathophysiology » et « renal dialysis ", et n'avons retenu que les études chez l'humain. Nous avons limité nos résultats aux dix

Tableau 1. Facteurs incriminés dans la genèse de l'HTA chez le dialysé

\footnotetext{
Action sur le débit cardiaque :

augmentation du volume extracellulaire

Action sur les grosses artères :

âge, calcifications

Action sur les petites artères et artérioles : déficit en oxyde nitrique excès en rénine-angiotensine excès en endothéline excès en catécholamines effet de l'érythropoiétine
}

dernières années. Ensuite, nous avons ajouté à cette liste des articles que nous avons jugés essentiels pour la compréhension de ce sujet.

Dix-neuf articles ont ainsi été trouvés, dont huit ont conclu que la surcharge hydrosodée était un facteur principal dans le développement de l'HTA en hémodialyse comme en dialyse péritonéale. Pour ce dernier traitement, la surcharge en volume est corrélée avec la TA surtout diastolique et une attention particulière à cette surcharge volumique permet de mieux contrôler le niveau de pression artérielle $[4,5]$.

Ce serait évidemment simpliste de conclure que le nombre de publications sur un thème est à lui seul décisif quant à son importance absolue ou relative. Cependant, le rôle majeur de la surcharge hydrosodée reste indiscutable (Fig. 1).

Deux des huit articles portant sur cette surcharge hydrosodée ont exploré son rôle par le recours à des études d'interventions [6,7]. Une ultrafiltration plus intense a eu pour conséquence une réduction significative de la tension artérielle systolique, de respectivement $7 \mathrm{mmHg}$ et $20 \mathrm{mmHg}$. L'étude de Fagugli [7] démontrait une réduction de la quantité d'eau extracellulaire de $4 \%$, avec diminution de l'utilisation d'antihypertenseurs et de la masse ventriculaire cardiaque. L'interprétation en a été simple - une diminution modeste du volume intravasculaire réduisait la tension artérielle.

Les six travaux restants ont testé la relation associative entre surcharge hydrosodée et HTA [8-13]. En particulier, l'analyse bioélectrique de l'impédance tissulaire a été utilisée, avec pour résultat de bonnes corrélations entre la quantité d'eau extracellulaire et la tension artérielle. La contribution de Katzarski a apporté aussi un plus dans la compréhension du phénomène. Ce groupe a comparé dialyse longue ou de durée habituelle. La conclusion essentielle fut que le contrôle du VEC pouvait normaliser la TA, d'une façon indépendante de l'efficacité ou la durée dialytique.

La combinaison d'effets du VEC et des résistances artérielles sur la TA pourrait cependant être en cause. Le rôle du neuropeptide $Y$ a été soutenu par Odar-Cederlof et al [9]. Pour ces auteurs, la concentration sérique de ce peptide vasoconstricteur augmente lors de l'expansion du VEC, potentialisant le rôle de la surcharge hydrosodée. Des travaux plus anciens ont incriminé un phénomène similaire. Il s'agit d'une action vasoconstrictrice de substances apparentées chimiquement à la digitaline et sécrétées en réponse à un excès de volume circulant [14]. Il est possible que de telles substances vasoconstrictrices expliquent le phénomène «lag » décrit par Charra au cours duquel sont séparés 


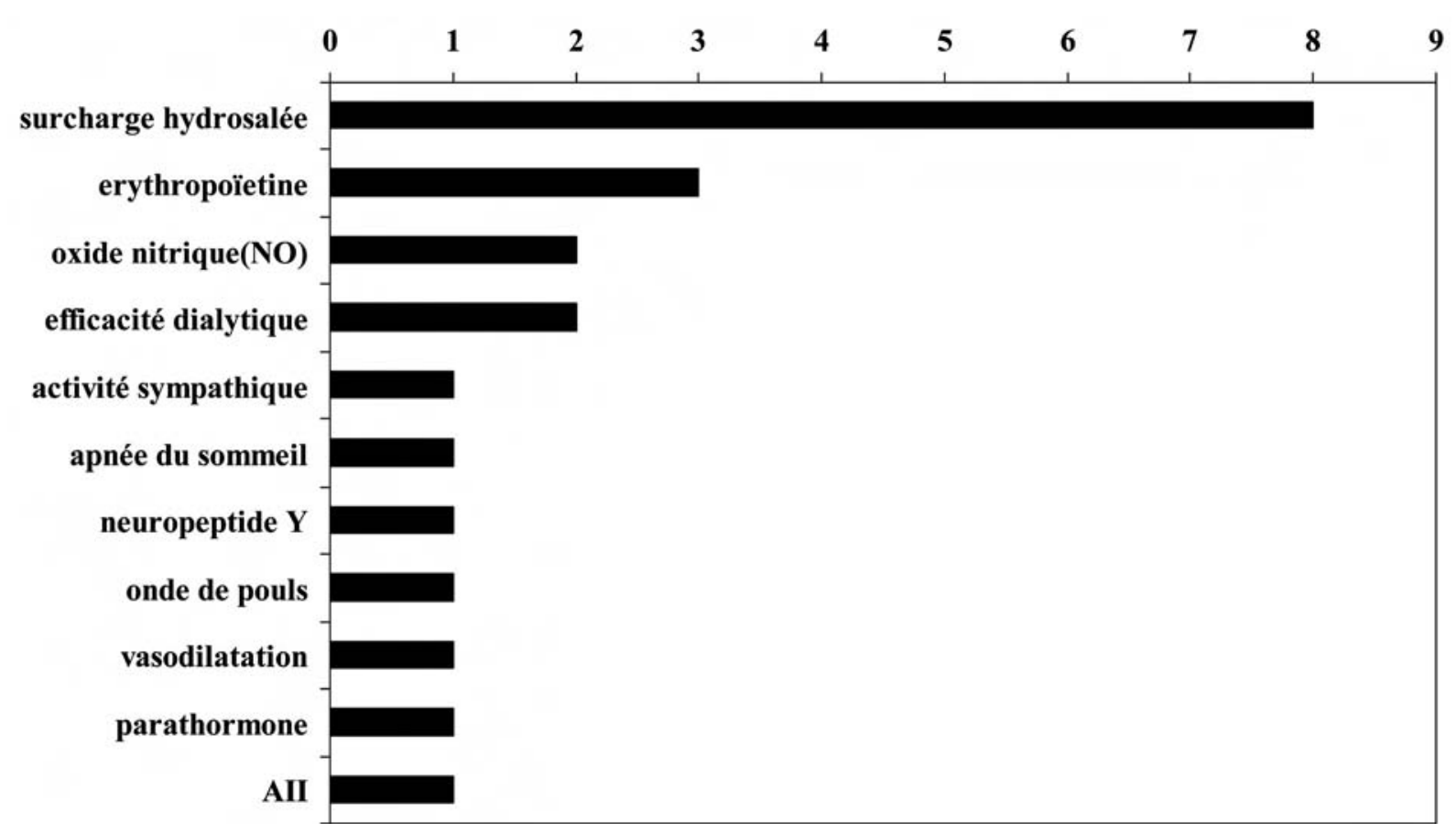

Figure 1. Nombre et sujet des articles ayant trait à la physiopathologie de l'HTA des dialysés, publiés entre 1997 et 2007 (6-24.) Le nombre cumulatif est plus grand que 19, car quelques articles discutent de plus qu'un seul facteur.

l'effet rapide sur la TA de la diminution du VEC et celui plus lent sur la TA lors du maintien du contrôle volumique. Rappelons ici les expériences de Guyton qui ont montré qu'une charge en chlorure sodique (et en eau) conduisait à une augmentation de TA, d'abord via une augmentation du VEC et du débit cardiaque et ensuite via celle des résistances vasculaires [15]. Secondairement, l'élévation de TA est source d'une natriurèse qui ramène cette TA à la normale. Cette relation «TA-diurèse » ne peut agir au stade de la dialyse.

Il faut aussi considérer l'impact, sur les résistances artérielles, de la modification de la concentration en sodium dans le dialysat [16]. Il est probable que des teneurs en sodium du dialysat plus élevées que celles du sérum puissent être à l'origine d'une induction osmotique de soif. Ceci aura tendance à augmenter le VEC. Mais cet effet du sodium dans le dialysat n'est pas aussi simple. Le bénéfice sur la TA d'un dialysat relativement hyponatrémique ( $137 \mathrm{mmol} / \mathrm{l})$ découlerait en partie d'un effet de diminution des résistances artérielles [17]. Dans une étude limitée à 9 patients (Tableau 2), nous avons pu montrer l'effet positif d'une diminution du sodium dans le dialysat, passant de 140 jusqu'à $136 \mathrm{mmol} / /$ sans changer le régime diététique ni le processus de dialyse. Il y a eu une chute progressive de la TA systolique, de $11 \mathrm{mmHg}$ sur les deux mois suivant ce changement. Quatre de ces patients affirmaient avoir eu moins soif, et deux ont pu arrêter leurs agents antihypertenseurs. Les poids secs ont augmenté en moyenne de 1,8 kg, ce qui suggère que la réduction de TA a été plus la conséquence d'une chute des résistances artérielles que d'une diminution du VEC.

Parmi les facteurs exogènes agissant sur la TA, l'érythropoïétine (EPO) a été impliquée, dès les premières années de son utilisation. Une méta-analyse récente confirme le risque d'une HTA mal contrôlée lorsque le taux d'hémoglobine est normalisé chez l'insuffisant rénal [18]. L'étude de Grekas rapporte même une corrélation identique entre HTA et

Tableau 2. Effet de la diminution de la concentration en sodium du dialysat

\begin{tabular}{lccc}
\hline Temps & TA syst. $\mathrm{mmHg}$ & Gain interdialytique, $\mathrm{kg}$ & Poids sec, kg \\
\hline Avant & 177 & 4,0 & 66,1 \\
Après & 166 & 3,6 & 67,9 \\
\hline
\end{tabular}


surcharge hydrosodée qu'entre HTA et dosage d'EPO utilisé [11]. Le mécanisme de cet effet hypertenseur de l'EPO passe pour certains par une vasoconstriction $[19,20]$. Cependant, l'influence exacerbante de l'EPO sur la TA n'est pas retrouvée par tous. Par mesure ambulatoire de la tension artérielle, Berns et al [21] n'ont pas observé d'HTA plus sévère sur un suivi d'un an chez des patients dialysés chez qui l'hématocrite était normalisé (hématocrite de $42 \%$ ).

D'autres mécanismes vasoconstricteurs ont été avancés. Trois groupes, mettent en cause un déficit en oxyde nitrique (NO) $[20,22,23]$. Le groupe d'Erkan [23] établit même une relation dose/ réponse inversée entre les taux pré-dialytiques de NO sanguin et la TA (Fig. 2).

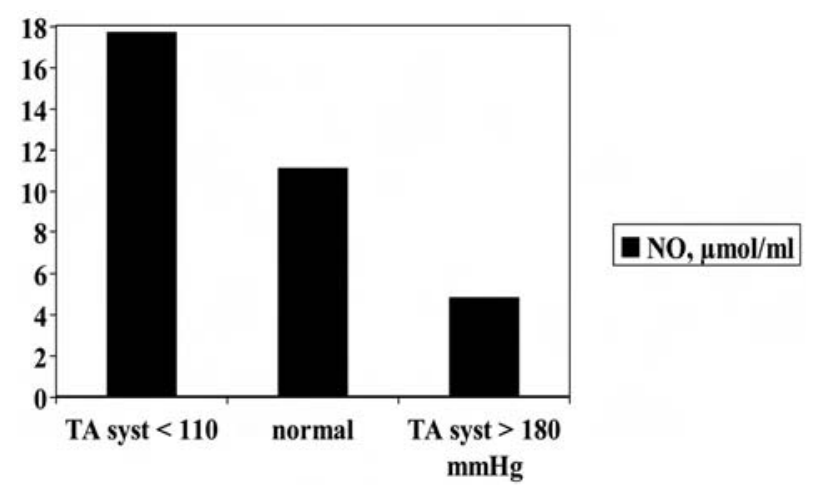

Figure 2. Relation entre les taux sanguins d'oxyde nitrique et la tension artérielle, selon Erkan et al. Les valeurs de TA de prédialyse rapportées sont soit normales, soit inférieures à $110 \mathrm{mmHg}$, soit supérieures à $180 \mathrm{mmHg}$.

Un polymorphisme de l'enzyme de la NO synthase endothéliale a été incriminé dans la relation TA et gain de poids interdialytique [22]. L'allèle « $a$ », qui se caractérise par une moindre production de NO, est associé à une augmentation interdialytique de la TA de $6 \mathrm{mmHg}$ supérieure à celle des patients ayant l'allèle « $b$ ». La montée de la TA lors du gain de poids semble être en partie déterminée par le NO ou plutôt sa carence liée à soit une enzyme déficiente, soit une diminution de production de NO par anomalie de substrat due à l'existence d'analogue circulant de l'arginine (« asymetrical dimethyl arginine ») chez le dialysé. Ceci permet de mieux comprendre le rapport de Farkas et al [24]. Ces auteurs montrent une réactivité microvasculaire altérée chez des patients en dialyse chronique. En comparaison avec des personnes normales, les sujets dialysés avaient une vasodilatation, sous l'influence d'acétylcholine perfusée mais aussi de nitroprussiate de sodium, diminuée de $50 \%$ au niveau de leur microcirculation, ainsi que des taux sanguins d'endothéline plus élevés. On peut donc intégrer cette vasodilatation diminuée au problème de la dysfonction endothéliale.

Un autre aspect vasculaire concerne le système nerveux sympathique.

Parmi les 19 articles de la figure 1, il n'y en a cependant qu'un seul centré spécifiquement sur l'activité de ce système sympathique, retrouvée augmentée au niveau musculaire, et ce en relation avec les reins natifs. Cette anomalie est notée chez des patients soit en dialyse soit greffés [25]. Du fait que la localisation anatomique de résistances artérielles se trouve dans les petites artères et artérioles de la circulation splanchnique et musculaire, on peut penser que cet effet sur les nerfs sympathiques contribue à l'HTA par vasoconstriction. Ce versant de la physiopathologie verra probablement un intérêt croissant, de par la découverte récente de la rénalase, enzyme rénale catabolisant les catécholamines mais déficiente chez des patients en dialyse chronique [26]. On peut proposer que les reins natifs de tels patients aient des effets néfastes à la fois par augmentation de l'activité nerveuse sympathique et par manque de catabolisme des catécholamines circulantes.

Dans cette optique, il faut aussi mentionner le problème de l'apnée du sommeil, rencontré quatre fois plus fréquemment chez le sujet en dialyse chronique que dans une population générale de même âge, sexe, index pondéral, ou race [27]. Les dangers de l'apnée du sommeil semblent découler d'une activité sympathique accrue, avec en plus un tonus vagal diminué [28]. Ces deux facteurs favoriseraient la mort subite en dialyse. La corrélation positive entre la présence d'une HTA en dialyse chronique et la sévérité de l'apnée du sommeil ne paraît pas dépendre du taux d'hémoglobine, du $\mathrm{Kt} / \mathrm{V}$, ou encore de l'index pondéral [29].

Les troubles du sommeil peuvent cependant être améliorés par une dialyse plus efficace [30], peutêtre par diminution de l'activité sympathique. Une diminution de la TA moyenne de 102 à $90 \mathrm{mmHg}$ a été notée après seulement deux mois de dialyse quotidienne, ayant augmenté l'efficacité dialytique d'un $\mathrm{Kt} / \mathrm{V}$ de 1,2 à 2 . Ceci a été mis en relation avec une diminution des taux plasmatiques de noradrénaline passant de $2,6 \mathrm{nmol} / \mathrm{l}$ à $2 \mathrm{nmol} / \mathrm{l}$, et des résistances périphériques réduites de 1967 dyne* $\mathrm{s}^{*} \mathrm{~cm}(-5)$ à 1499 dyne* $\mathrm{s}^{*} \mathrm{~cm}(-5)$ [31]. L'ensemble soutient l'idée de résistances artérielles accrues chez les personnes en dialyse chronique, surtout celles avec efficacité dialytique marginale, et partiellement réversible par une augmentation 
de cette efficacité. Celle-ci, appréciée par un Kt/ $V$ (urée) passant de 0,9 à 1,6, a été associée à une diminution de la TA systolique de 147 à $133 \mathrm{mmHg}$ [32]. Cependant, comme déjà rappelé plus haut, le contrôle du VEC est interprété par certains comme plus important que l'efficacité dialytique. Les deux aspects, volume et résistances artérielles, jouent donc des rôles mutuellement renforçants dans la physiopathologie de l'HTA en dialyse.

Comme aussi déjà mentionné, l'HTA systolique est fréquente au stade de la dialyse. Que l'onde de pouls soit corrélée avec l'HTA systolique n'est pas surprenant [33]. Ceci pourrait avoir un lien bidirectionnel, la TA systolique favorisant les remaniements de la paroi des grandes artères, et ceux-ci, par l'effet de réduction de l'élasticité vasculaire, étant à l'origine d'une augmentation de la TA systolique. Chen et al montrent que la sensibilité au volume de la TA en dialyse augmente avec la diminution de la compliance ventriculo-artérielle [34]. Il est aussi utile de rappeler que les modalités de dialyse plus intense, telles que la dialyse quotidienne, ont été associées à des effets bénéfiques sur l'onde de pouls.

Parmi les dix-neuf articles de notre enquête sur Medline, il n'y en a qu'un seul sur l'angiotensine II [35]. Dans celui-ci a été exploré le rôle possible de l'angiotensine II dans la genèse de l'HTA chez des enfants anéphriques en dialyse chronique. Aucune relation n'a pu être trouvée. Shin et collègues rapportent, de leur côté, une corrélation significative entre rénine plasmatique et TA chez les sujets dialysés depuis moins de dix ans, mais une corrélation inverse chez ceux en dialyse pour une durée plus longue [36]. Grekas et collègues ont aussi montré des activités plasmatiques de rénine plus élevées chez des dialysés hypertendus [37]. La diminution de la TA après bi-néphrectomie pourrait en partie s'expliquer par réduction du système rénine-angiotensine, sans pour autant que l'HTA habituelle des dialysés soit en étroite relation avec ce système.

Pour ce qui est de la calcémie comme cause d'HTA en dialyse, l'absence d'influence de la parathyroïdectomie sur l'HTA [38] ne diminue pas l'importance jouée par le calcium. L'hypercalcémie peut toujours être à l'origine d'une augmentation de la TA, et nous avons par ailleurs déjà cité ta notion de l'effet hypertenseur de l'EPO, action médiée par une augmentation du calcium intracellulaire [19].

\section{Conclusions et suggestions pour de futurs travaux}

L'HTA chez le patient dialysé est présente dès l'initiation du traitement et reste fréquente malgré celui-ci, jouant un rôle dans le risque cardio-vasculaire.

Sa physiopathologie est multifactorielle, expliquant peut-être les difficultés dans sa correction. Un excès de VEC joue un rôle majeur, favorisé par l'oligo-anurie des patients, l'apport sodé alimentaire et via le bain de dialyse. L'hyperactivité sympathique, la dysfonction endothéliale, la correction rapide de l'hématocrite par l'EPO, l'hypercalcémie et l'altération des grosses artères participent à la difficulté du contrôle de la TA par l'ultrafiltration majorée. Le fait que la dialyse quotidienne ou nocturne de longue durée améliore le niveau de TA s'explique par un retrait plus adéquat de facteurs vasoconstricteurs outre la facilité du contrôle volumique.

Des publications fascinantes mettent en cause un déficit artériolocapillaire comme facteur de risque pour l'hypertension, c'est à dire un simple déficit anatomique du nombre de vaisseaux de type résistance $[39,40]$. On peut se demander si ce déficit est en jeu chez les insuffisants rénaux, et si oui, quelle doit en être l'approche thérapeutique optimale.

\section{Références}

[1] Buckalew VM, Berg RL, Wang SR, Porush JG, Rauch S, Schulman G, et al. Prevalence of hypertension in 1795 subjects with chronic renal disease: the modification of diet in renal disease study baseline cohort. Am J Kidney Dis 1996;28:811-21.

[2] Agarwal R, Nissenson AR, Batlle D, Coyne DW, Trout JR, Warnock DG. Prevalence, treatment, and control of hypertension in chronic hemodialysis patients in the United States. Am J Med 2003;115:291-7.

[3] Agarwal R, Lewis RR. Prediction of hypertension in chronic hemodialysis patients. Kidney Int 2001;60:1982-9.

[4] Wang X, Axelsson J, Lindholm B, Wang T. Volume status and blood pressure in continuous ambulatory peritoneal dialysis patients. Blood Purif 2005;23:373-8.

[5] Konings CJ, Kooman JP, Schonck M, Dammers R, Cheriex E, Palmans-Meulemans AP, et al. Fluid status, blood pressure, and cardiovascular abnormalities in patients on peritoneal dialysis. Perit Dial Int 2002;22:477-87.

[6] Kursat S, Aysel S, Alici T, Tezcan UK. Blood pressure and ejection fraction changes due to ultrafiltration in hemodialysis. J Nephrol 2006;19:84-90.

[7] Fagugli RM, Pasini P, Pasticci F, Ciao G, Cicconi B, Buoncristiani U. Effects of short daily hemodialysis and extended standard hemodialysis on blood pressure and cardiac hypertrophy: a comparative study. J Nephrol 2006;19:77-83.

[8] Dionisio P, Valenti M, Bergia R, Caramello E, Stramignioni $E$, Berto IM, et al. Influence of the hydration state on blood pressure values in a group of patients on regular maintenance hemodialysis. Blood Purif 1997;15:25-33.

[9] Odar-Cederlof I, Ericsson F, Theodorsson E, Kjellstrand CM. Is neuropeptide $Y$ a contributor to volume-induced hypertension? Am J Kidney Dis 1998;31:803-8. 
[10] Katzarski KS, Charra B, Luik AJ, Nisell J, Filho JCD, Leypoldt JK, et al. Fluid state and blood pressure control in patients treated with long and short haemodialysis. Nephrol Dial Transpl 1999;14:369-75.

[11] Grekas D, Barmichas G, Bacharaki D, Goutzaridis N, Kasimatis E, Tourkantonis A. Hypertension in chronic haemodialysis patients:current view on pathophysiology and treatment. Nephrol 2000;53:164-8.

[12] Alvarez-Lara MA, Martin-Malo A, Espinosa M, RodriguezBenot A, Aljama P. Blood pressure and body water distribution in chronic renal failure patients. Nephrol Dial Transplant 2001;16 Suppl 1:94-7.

[13] Fagugli RM, Pasini P, Quintaliani G, Pasticci F, Ciao G, Cicconi $B$, et al. Association between extracellular water, left ventricular mass and hypertension in hemodialysis patients. Nephrol Dial Transplant 2003;18:2332-8.

[14] Krzesinski JM, Godon JP, Rorive G. Erythrocyte sodiumpotassium activities, plasma natriuretic activity, and peripheral vascular resistances during hemodialysis or hemofiltration. J Clin Hypertens 1985;3:245-56.

[15] Guyton A. Renal functional curve:a key to understanding the pathogenesis of hypertension? Hypertension 1987; 10:1-6.

[16] Krautzig S, Janssen U, Koch K, Granolleras C, Shaldon S. Dietary salt restriction and reduction of dialysate sodium to control hypertension in maintenance hemodialysis patients. Nephrol Dial Transplant 1998;13:552-553.

[17] De Paula F, Peixoto AJ, Pinto LV, Dorigo D, Patricio PJM, Santos SFF. Clinical consequences of an individualized dialysate sodium prescription in hemodialysis patients. Kidney Int 2004;66:1232-1238.

[18] Phromintikkul A, Haas SJ, Elsik M, Krum H. Mortality and target haemoglobin concentrations in anaemic patients with chronic kidney disease treated with erythropoietin:a meta-analysis. Lancet 2007;369:381-88.

[19] Schiffl H, Lang SM. Hypertension induced by recombinant human erythropoietin can be prevented by indomethacin. Pathogenetic role of cytosolic calcium. Eur J Med Res 1997;2:97-100.

[20] Shimada N, Saka S, Sekizuka K, Tanaka A, Takahashi Y, Nakamura $T$, et al. Increased endothelin:nitric oxide ratio is associated with erythropoietin induced hypertension in hemodialysis patients. Renal Failure 2003;25:569-78.

[21] Berns JS, Rudnick MR, Cohen RM, Bower JD, Wood BC. Effects of normal hematocrit on ambulatory blood pressure in epoetin-treated hemodialysis patients with cardiac disease. Kidney Int 1999;56:253-260.

[22] Yokoyama K, Tsukada T, Nakayama M, Hara S, Yamada A, Kawaguchi $Y$, et al. An intron 4 gene polymorphism in endothelial cell nitric oxide synthase might modulate volume dependant hypertension in patients on hemodialysis. Nephron 2000;85:232-7.

[23] Erkan E, Devarajan P, Kaskel F. Role of nitric oxide, endothelin 1, and inflammatory cytokines in blood pressure regulation in hemodialysis patients. Am J Kidney Dis 2002;40:76-81.

[24] Farkas K, Nemcsik J, Kolossvary E, Jarai Z, Nadory E, Farsang $C$, et al. Impairment of skin microvascular reactivity in hypertension and uremia. Nephrol Dial Transplant 2005;20:1821-7.
[25] Hausberg M, Kosch M, Harmelink P, Barenbrock M, Hohage H, Kisters $\mathrm{K}$, et al. Sympathetic nerve activity in end-stage renal disease. Circulation 2002;106:1974-9

[26] Xu J, Li G, Wang P, Velazquez H, Yao X, Li Y, et al. Renalase is a novel, soluble monoamine oxidase that regulates cardiac function and blood pressure. J Clin Invest 2005;115:1275-80.

[27] Unruh ML, Sanders MH, Redline S, Piraino BM, Umans JG, Hammond TC, et al. Sleep apnea in patients on conventional thrice weekly hemodialysis: comparison with matched controls from the sleep heart health study. J Am Soc Nephrol 2006;17:3503-9.

[28] Hilton MF, Chappell MJ, Bartlett WA, Malhotra A, Beattie JM, Cayton RM. The sleep apnoea/hypopnoea syndrome depresses waking vagal tone independent of sympathetic activation. Eur Resp J 2001;17:1258-66.

[29] De Oliveira Rodrigues CJ, Marson O, Tufic S, Kohlmann O, Guimarães SM, Togeiro S, et al.Relationship among endstage renal disease, hypertension, and sleep apnea in nondiabetic dialysis patients. Am J Hypertension 2005;18: 152-7.

[30] Hanly PJ, Pierratos A. Improvement of sleep apnea in patients with chronic renal failure who undergo nocturnal hemodialysis. N Engl J Med 2001;344:102-7.

[31] Chan CT, Harvey PJ, Picton P, Pierratos A, Miller JA, Floras JS. Short term blood pressure, noradrenergic, and vascular effects of nocturnal home hemodialysis .Hypertension 2003;42:925-31.

[32] Panagoutsos SA, Yannatos EV, Passadakis PS, Thodis ED, Galtsidopoulos OG, Vargemezis VA. Effects of hemodialysis dose on anemia, hypertension and nutrition. Renal Failure 2002;24:615-21.

[33] Wang MC, Tsai WC, Chen JY, Huang JJ. Stepwise increase in arterial stiffness corresponding with the stages of chronic kidney disease. Am J Kidney Disease 2005;45:494-501.

[34] Chen CH, Lin YP, Yu WC, Yang WC, Ding YA. Volume status and blood pressure during long term hemodialysis. Hypertension 2003;42:257-62.

[35] Seguias L, Zilleruelo G, Strauss J, Abitbol C, Montane B. Angiotensin II and endothelin 1 levels in children with renoprival hypertension. Ped Nephrol 2001;16:493-6.

[36] Shin J, Sakai R, Nishioka M, Okudaira H, Arakawa T, Fujita Y, et al. Blood pressure and renin in long term hemodialyis patients. Nippon Jinzo Gakkai Shi 1998;38:13-21.

[37] Grekas D, Kalevrososglou I, Karamouzis M, Geropoulou E, Kabouris $\mathrm{H}$, Tourkantonis A. Effect of sympathetic and plasma renin activity on hemodialysis hypertension. Clin Nephrol 2001;55:115-20.

[38] Ifudu O, Matthew JJ, Macey LJ, Hong LJ, Sumrani N, Sommer BG, et al. Parathyroidectomy does not correct hypertension in patients on maintenance hemodialysis. Am J Nephrol 1998;18:28-34.

[39] Noon JP, Walker BR, Webb DJ, Shore AC, Holton DW, Edwards HV, et al. Impaired microvascular dilatation and capillary rarefaction in young adults with a predisposition to high blood pressure. J Clin Invest 1997;99: 1873-9.

[40] Levy BI, Ambrosio G, Pries AR, Struijker-Boudier HAJ. Microcirculation in hypertension: a new target for treatment? Circulation 2001;104:735-40. 